\title{
Vitamin D and non-alcoholic fatty liver disease: a systematic review and meta-analysis
}

\author{
O.A. Akinyemi ${ }^{1}$, S.A. Lanham-New ${ }^{1}$ and A.L. Darling ${ }^{1}$ \\ ${ }^{1}$ Department of Nutritional Sciences, School of Biosciences and Medicine, Faculty of Health and Medical Sciences, \\ University of Surrey, Guildford, GU2 $7 X H$.
}

Non-Alcoholic Fatty Liver Disease (NAFLD) describes a wide range of hepatic pathological conditions beginning with accumulation of lipids, especially triglycerides, in the hepatocytes in the absence of significant alcohol intake ${ }^{1}$. NAFLD shows a strong association with obesity, type 2 diabetes (T2D), insulin resistance (IR), hyperlipidaemia and arterial hypertension. Hence it is referred to as the hepatic manifestation of the metabolic syndrome ${ }^{2}$. Studies have shown significant inverse associations between 25 -hydroxyvitamin D $(25(\mathrm{OH}) \mathrm{D})$ concentration and other diseases including diabetes, hyperlipidaemia, hypertension and peripheral vascular disease, suggesting a possible role for vitamin $\mathrm{D}$ in the pathogenesis of NAFLD ${ }^{3,4}$. The aim of this study was to systematically review the association between NAFLD and vitamin D using data from both randomised control trials (RCT), cross-sectional and case-control studies. This was to quantify differences in $25(\mathrm{OH}) \mathrm{D}$ status between individuals with and without NAFLD, as well as the effect of vitamin D supplementation in NAFLD patients on metabolic function.

The PUBMED database was electronically searched for relevant studies in adolescents and adults from inception up until April 2017. A total of 129 relevant studies were identified and 24 of these were suitable for inclusion in the systematic review.

Meta-analysis of cross-sectional, cohort and case control studies suggested a statistically significantly lower serum $25(\mathrm{OH})$ in those with NAFLD, compared with those without NAFLD (Mean Difference ${ }_{\text {(random) }}=-16.80 \mathrm{nmol} / \mathrm{L}[-24.38,-9.21] \mathrm{I}^{2}=96 \% \mathrm{P}<0.0001$ ). In addition, meta-analysis of 4 randomised control trials (RCTs) suggested a statistically significant effect of vitamin D supplementation on serum $25(\mathrm{OH})$ D levels (Mean Difference $(\mathrm{random})=53.08 \mathrm{nmol} / \mathrm{L}[28.22,77.93] \mathrm{I}^{2}=96 \% \mathrm{P}<0.0001$ ), Furthermore, meta-analysis of HOMA-IR suggested no statistically significant effect of vitamin D supplementation (Mean Difference $\left(\right.$ random) $=-0.26[-2.02,1.51] \mathrm{I}^{2}=$ $99 \% \mathrm{P}=0.78$ ).

In conclusion, 25(OH)D status was lower in individuals with NAFLD than in those without NAFLD, suggesting that serum 25 $(\mathrm{OH}) \mathrm{D}$ levels may be a factor in the development of NAFLD. However, it may also simply be a result of persons with NAFLD having higher adiposity than those without NAFLD, as increased adiposity is associated with reduced $25(\mathrm{OH}) \mathrm{D}$ concentration. This is the first study to report a meta-analysis of vitamin D supplementation in NAFLD patients. No effect was found of vitamin D supplementation on HOMA-IR. Therefore this review does not support the use of vitamin D supplementation for NAFLD patients in terms of improving metabolic function, although it could have other health benefits, such as for immune and musculoskeletal health.

1. Sanyal et al., 2010. $N$ Engl J Med, 362, 1675-85.

2. Loomba et al., 2012. Hepatol, 56,943-51.

3. Anderson et al., 2010. Am J cardiol, 106,963-68.

4. Li et al., 2013. Endocrine, 44,465-72. 